\title{
Multi-Modal Case Study on MRI Brain Tumor Detection Using Support Vector Machine, Random Forest, Decision Tree, K-Nearest Neighbor, Temporal Convolution \& Transfer Learning
}

\author{
Partha Sutradhar, Prosenjit Kumer Tarefder, Imran Prodan, Md. Sheikh Saddi, Victor Stany Rozario
}

\begin{abstract}
In the Medical field, Brain Tumor Detection has become critical and demanding task because of their several shapes, locations, and the intensity of image. That's why an automated system is important to aid physicians and radiologists in detecting and classifying brain tumor. In this research, we have discussed different machine learning as well as deep learning algorithm which are mostly used for image classification. We have also compared different models that are being used for tumor classification based on machine learning and deep learning. MRI images of Glioma tumor, Pituitary tumor, Meningioma tumor are the base of this research, and we have compared different techniques along with the accuracy of different classification model using those MRI images. We have used different deep learning pre-trained model for training brain tumor images. Those pre-trained models have provided outstanding performance along with less power consumption and computational time. EfficientNet-B3 has provided the best accuracy of $98.16 \%$ among other models as well as traditional machine learning algorithms. The experimental result of this model is proven the best and most efficient for tumor detection and classification in comparison with other recent studies.
\end{abstract}

Index Terms - Random Forest (RF), Support Vector Machine (SVM), Decision Tree (DT), Temporal Convolutional Network (TCN), K-Nearest Neighbor (KNN), Convolutional Neural Network (CNN), Brain Tumor Detection, Transfer Learning.

\section{INTRODUCTION}

B rain tumor can be described as uncontrolled and unnatural growth of cells in brain. The uncontrolled growth of these cells within a certain part of the brain causes

Partha Sutradhar is a BSc student of Computer Science Engineering department from American International University-Bangladesh, Dhaka-1229. Email: partharaj.dev@gmail.com.

Prosenjit Kumer Tarefder is a BSc student of Computer Science Engineering department from American International University-Bangladesh. Email: prosenjittarefder@gmail.com.

Imran Prodan is a BSc student of Computer Science Engineering department from American International University-Bangladesh. Email: prodhanimran45@gmail.com.

Md. Sheikh Saddi is a BSc student of Computer Science Engineering department from American International University-Bangladesh. Email: sheikhsaddi1679@gmail.com.

Victor Stany Rozario is an Assistant Professor of the Department of Computer Science, Faculty of Science and Technology, American International University-Bangladesh, Dhaka-1229, Bangladesh.

Email: stany@aiub.edu. death of a person if not detected earlier. Some tumors are cancerous thus they needed to be detected earlier before they spread too much on the brain. People are suffering from it without realizing the great danger of its growth. Better MRI analysis of tumor images is now a strong research area in the medical field. The examination of these brain tumors are based on imaging the data analysis of MRI images. The MRI images are found very clear and precise. MRI images are needed to be recognized in the early stage of the tumor before they spread too much.

There are different types of brain tumor that has been detected in human brain. Glioma tumor, Pituitary tumor, Meningioma tumor are the main concern tumor of this paper. Usually, Glioma tumor which is occurred in the brain as well as spinal cord. Among all of the brain tumors, Glioma tumor occurs most which is around 33\%. This tumor is arised from a supportive cell called Gluey which is surrounded by the nerve cells. From that gluey cell, Glioma tumors can affect the brain [32]. Pituitary tumor is another type of tumor which starts growing in the gland cells. This tumor is basically small gland located inside the skull, just under the brain and over nasal passages. Pituitary tumors are around $10 \%$ of brain tumor which grows slowly [33]. Meningioma is mostly common brain tumor which is around $30 \%$ of all tumors occurred in the brain. It originates from the three outer layers of brain cells known as meninges which is covered and protected under the skull. Meningioma is a slow growth tumor and around $85 \%$ of them are noncancerous [32].

The recent past few years, we have seen an explosive amount of growth in Artificial Intelligence, Machine Learning, Data Mining, and Data Science. Traditional Machine Learning algorithms like Support Vector Machine (SVM), K-Nearest Neighbor (KNN), and Random Forest (RF) were really great in the old days of data analysis sector. But in the modern days Artificial Intelligence, Computer Vision and Deep Learning algorithms have performed far better than the traditional machine learning algorithms. Here, in this paper we are going to make comparative result analysis of Machine Learning and Deep Learning models and seek for better classifier or model which is appropriate for Brain Tumor Classification. 


\section{LITERATURE REVIEW}

A brain tumor has become a major issue around the world as many people are affecting and dying every day. So, it is very much important to discover either tumor is a cancerous tumor or not. If the Brain tumor is detected earlier, then actual situation of the tumor can be found and effective procedure can be taken to prevent the growth of the tumor. In this research, we discuss many pattern recognition algorithms for machine learning [1]. We have compared various machine learning \& deep learning algorithms and based on that we finalize K-Nearest Neighbor (KNN), Support Vector Machine (SVM), Random Forest (RF), Decision Tree (DT), and Convolutional Neural Network $(\mathrm{CNN})$. We try to find the fine-tuned algorithm that may deliver the best outcome for medical treatment and diagnostics after successfully experimenting with all of these algorithms. By comparing all of those algorithms, we may achieve the best image pattern recognition algorithm that will give an accurate result in medical treatment and diagnosis.

M. Sajjad et al suggested a method using deep learning technique to summarize brain tumors into multiclass and multiple grades [2]. At different stages of brain tumors, transfer learning has used for the classification of the tumors. The MRI image was passed into the Convolutional Neural Networks (CNN) model to predict outcome of the brain tumor. Before and after image augmentation, two different datasets Brain Tumor and Radiopaedia were used for this research analysis. The model obtained around $90.67 \%$ accuracy with image augmented data and without image augmentation $87.38 \%$.

G. Singh et al [7] proposed a method that includes normalization and division of K-Means. This process eliminates unwanted noise from input MRI images. Multiple filters are being used for an MRI image to reduce noise from MRI images and normalize the pre-processed image for MRI classification. Finally, the K-Means algorithm usually divides the image and removes the tumor from the MRI. Naïve Bayes and Support Vector Machine (SVM) algorithms are applied to classify the magnetic resonance and provide clear estimates. The naïve compilations of Bayes and SVM delivered 87.23\% and $91.49 \%$ accuracy, respectively.

The goal of the research is to determine which algorithm or classifier works best for MRI image classification using Machine Learning or Deep Learning. Machine learning methods are suitable for small data and have limits on tuning capabilities. However, deep learning is suitable for massive data and can be tuned with various hyper-parameter optimization in the training phase. These algorithms are appropriate for classification and the other performs better in feature transformation. Therefore, our study is to compare different algorithms or classifier for brain tumor detection based on MRI images for identifying the best classifier.

\section{A. Support Vector Machine}

Support Vector Machine (SVM) used in supervised machine learning problems that are mostly used for regression, classification, and outlier detection. The main theory depends on a supervised learning mechanism [10]. This algorithm was introduced in 1963 by Lerner and Vapnik. SVM is a binary classifier based on a supervised learning mechanism. Support Vector Machine (SVM) operates in various types of data mining techniques, and machine learning. SVM algorithm works by creating a decision boundary which can separate $n$ dimensional space in the direction of different classes so that data points of different classes will represent the different category. Decision boundary is well-known as hyperplane which splits the datasets into different classes which are generated iteratively for minimizing errors. Two types of SVM algorithms are: a) Linear SVM, b) Non-linear SVM. Linear SVM means the linear separation of the linear dataset using one straight line. In the non-linear case, the separation of the non-linear dataset can't be done using a simple straight forward line [11].

Linear SVM does not just only focus on creating one hyperplane, also creates two parallel hyperplanes in such a manner that one of the hyperplanes will be passing to the nearest positive point and the other hyperplane will be passing to the nearest negative point. When computing this distance that is $\mathrm{d}+$ and $\mathrm{d}$ - the whole distance is called marginal distance. The main aim is to maximize this particular marginal plane. Based on those hyperplanes should be selected which have the maximum marginal distance. Concerning the marginal planes that are created the positive and negative points, the higher the marginal distance the more generalized the model is.

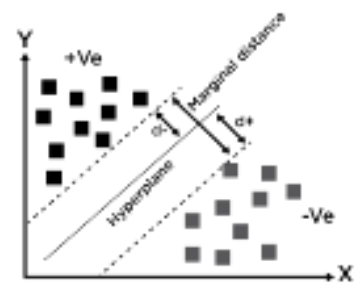

Fig. 1. Linear Separation

For Non-linear separation, the SVM kernel tries to convert the low dimension (2D) into high dimension (3D). After converting into the high dimension, a hyperplane is created which classifies these particular points. Concerning the hyperplane, a marginal plane will be found.

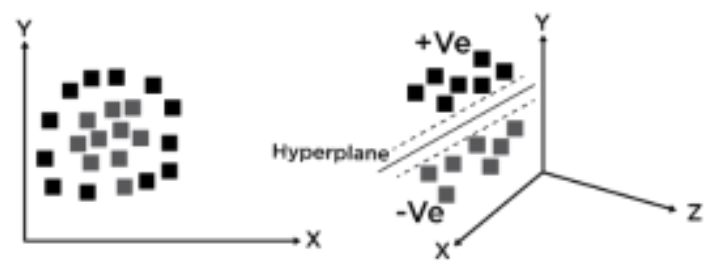

Fig. 2. Non-Linear Separation 
Here, Non-Linear Support Vector Machine (SVM) is important for our classifying task. Because, in linear Support Vector Machine (SVM) we can classify only two levels. But here, In Fig. 2 classification of non-linear Support Vector Machine (SVM) can classify more than two levels. In Fig. 2 we can see that, it can do multi class classification because of its non-linear property.

\section{B. Decision Tree}

Decision Tree also known as prognostic learning technique used for multiple classifications and regression problems. The decision tree is an Id3 algorithm that is repeatedly applied in data mining. Decision Tree means we will be constructing a kind of tree based on the attributes and finally after constructing the tree, we are going to see the leaf node as a final class labels. In this approach greedy algorithm works [13]. This algorithm applies top-down recursive and also divide-and-conquer rules. Decision tree is created by disjointing the source feature set. Here, feature with maximum value information gain will be in the root node and the other is going to be the leaf node of the base root node. Mathematical terms of decision tree described as:

$$
(\mathrm{x}, \mathrm{Y})=(\mathrm{x} 1, \mathrm{x} 2, \mathrm{x} 3, \ldots ., \mathrm{x} \mathrm{N}, \mathrm{Y})
$$

The relative variable is the output, we use output variable $\mathrm{Y}$ to forecast. The vector $\mathrm{x}$ contains input features. In this case, features are going to be the input of vector $x$ of MRI images and $\mathrm{Y}$ is the target value. Decision Tree classifier will be utilized to classify MRI as Pituitary Tumor, Meningioma Tumor, Glioma Tumor MRI images. In a Decision Tree, low bias provides less error by creating a tree to its complete depth results in over fitting.

1. Information Gain:

$$
\begin{aligned}
& \operatorname{Gain}(\mathrm{S}, \mathrm{A})=\text { Entropy }(\mathrm{S})-\sum \frac{\| S i \mid}{\|S\|} * \text { Entropy }(\mathrm{si}) \\
& \mathrm{S}=\text { Samples } \\
& \mathrm{A}=\text { Attribute } \\
& \mathrm{S}_{\mathrm{i}}=\text { Indivual Sample }[12]
\end{aligned}
$$

2. Entropy:

$$
\text { Entropy }=-\mathrm{P}(+) \log _{2} \mathrm{P}(+)-\mathrm{P}(-) \log _{2} \mathrm{P}(-)
$$

\section{Random Forest}

Simply, Random forests use ensemble machine learning techniques not only for classification but also for regression problems. Ensemble denotes combining several models and getting the output result of multiple models after training. Random forests are also called bootstrap aggregation. Random forest uses multiple Decision Tree and every Decision Tree is computed individually [9]. Some data sample from dataset is randomly distributed with every decision tree for training and find out every decision tree's mean accuracy. The mean value of these continuous values is taken from Bootstrap with the replacement technique. In Decision tree low bias means training is much better. Thus, Decision Tree gets properly trained for our training data set and training error will very less in this term. The High variance occurs when we get our new test data those decision trees are prone to give a larger number of errors. So, Random Forest is very good at these to produce low variance as the sample dataset gets split randomly at the time of training [8]. Random forest algorithms use multiple trees to minimize the danger of over fitting also this algorithm needs less time in the training phase. For a large dataset, this algorithm runs efficiently. It produces a highly accurate prediction. However, if a large quantity of data is missing this algorithm still can maintain accuracy.

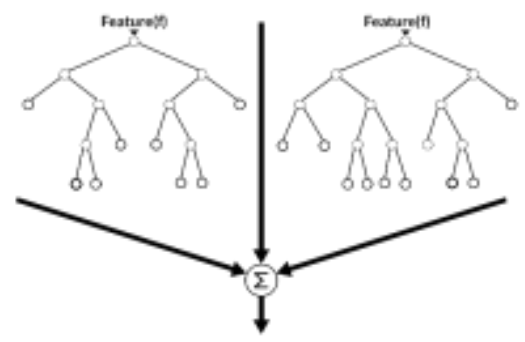

Fig. 3. Random Forest [31]

\section{K-Nearest Neighbor}

$\mathrm{K}$-nearest neighbor is a fundamental place to start in machine learning. It is the basis of a lot of things and the reason behind that it is very much easy to understand and incorporate it in other forms of machine learning. KNN is considered as the simplest supervised learning algorithms primarily used for classification which classifies an input data point depending on how that data point marks its neighbors. It is a wonderful algorithm used for solving nonlinear classified data points. KNN stores all existing cases and measures new cases on an equal basis. KNN algorithm is constructed on feature similarity [3]. In $\mathrm{KNN}, \mathrm{k}$ is a perimeter that denotes the value of nearest neighbors in such a way that it can contain the value in the majority utilizing the voting procedure. Picking the accurate $\mathrm{k}$ value is a procedure known as parameter tuning as well as is essential for enhanced accuracy. In all machine learning choosing the factor of $\mathrm{k}$ is very much tough work. Because, if the quantity of $\mathrm{k}$ is too low then bias gets too noisy and might not get the expected answer. The opposite way, if the quantity of $\mathrm{k}$ is too big then it is going to take forever to process. Because of that, the system will be stuck into processing issues and resource issues. To choose a value of $\mathrm{k}$ the formula is,

$$
\mathrm{K}=\sqrt{n}
$$

Where $\mathrm{n}$ denotes the entire number of values. If the $\mathrm{k}$ value happens to be an even number, then try to make it odd because it is better as well as it helps to avoid misperception between two classes [4]. KNN can be used when data is labeled, data sets are small, and noise-free. KNN is a lazy learner which doesn't learn any discriminative function via the given training set. If data are very complicated and the amount of data is very large, then $\mathrm{KNN}$ is not a suitable algorithm. To find the nearest neighbors, we will compute the Euclidean distance. Euclidean distance formula defines distance between two distinct points in a plane with coordinates $(\mathrm{x}, \mathrm{y})$ and $(\mathrm{a}, \mathrm{b})$ is given by, 
Distance $(\mathrm{d})=\sqrt{(x-a) 2+(y-b) 2}$

By using this formula, we will calculate Euclidean distance of unfamiliar data point form all the points in the dataset and using the Euclidean distance the closest neighbors is found [5]. In the KNN algorithm, $\mathrm{K}$ value selection is a very much crucial part and there exists no particular way to define the finest value for K. So, while testing, we need to try distinct $\mathrm{K}$ values to find the accurate and more suitable value. If we take a larger $\mathrm{K}$ value, that may occur many difficulties while prediction processing like higher complexity, more prediction time, more computation cost.

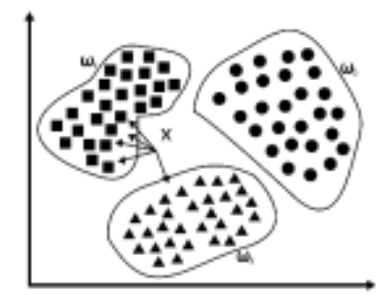

Fig. 4. K-Nearest Neighbor [30]

\section{E. Convolutional Neural Networks}

Well in this modern era of artificial intelligence, Convolutional Neural Network is an efficient type of deep learning method for image classification currently used in the industry for MRI images and many other classification task problems. Convolutional Layer work is to find out important feature maps to extract information from the images using filters to provide feature maps from given MRI Brain Tumor images. Convolutional Neural Network uses different type's filters to extract information and feature maps from an image. These filters collect the valuable informational feature maps from a given input image and collect valuable information then forward them to the Fully Connected Network (FCN) Layer for training the model. The more filters give us more feature maps, thus the network's model accuracy becomes better at recognizing patterns for undetected images [21]. There are many filters in CNN like sharping an image, horizontal and vertical filters, edge detection filters, Sobel filters, Laplace filters, etc. These filters help us detect images more accurately. Before forwarding the feature map in FCN layer $\operatorname{ReLu}$, the $\mathrm{F}(\mathrm{z})=\max (0, \mathrm{z})$ activation function is used to replace the negative values with zero from feature maps.

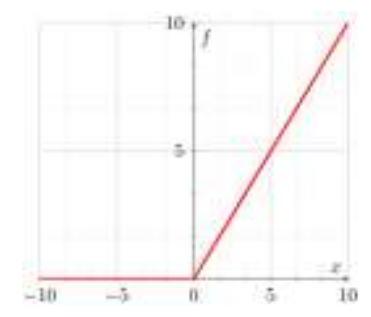

Fig. 5. ReLu [29]

$\mathrm{ReLu}$ is a popular fine-tuned activation function for deep learning and convolutional neural networks. ReLu activation function is short for a rectified linear unit [18]. Let's say we have a situation like $F(-0.56)=\max (0,-0.56)=0$ (Fig. 5). Now, y-axis value becomes zero and if we have a situation like this $\mathrm{F}(1.01)=\max (0,1.01)$, the corresponding to $\mathrm{y}$-axis value would be 1.01 in that case. ReLu is an efficient activation function that computes faster than a traditional sigmoid function and its derivatives can be easily found [20]. There are some advantages and disadvantages of this activation function. $\mathrm{ReLu}$ is great for solving vanishing gradient problems and faster to compute. Oppositely, ReLu tends to explode the activation function which makes training inaccurate or sometimes crashes the training and if most of the activations get value lower than zero then it leads to dying ReLu problem.

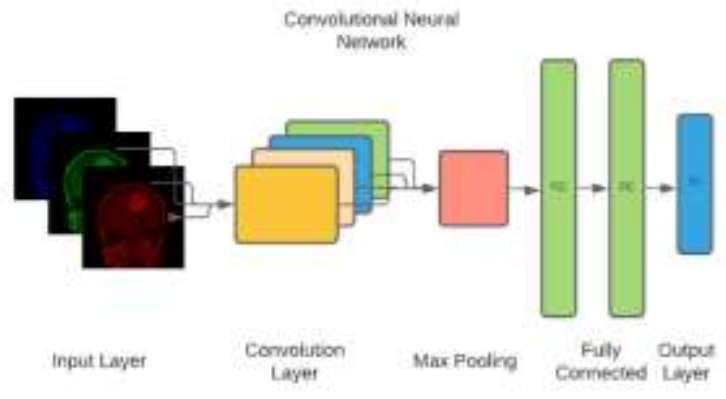

Fig. 6. Convolutional Neural Networks

Looking at the Convolutional Neural Networks in Fig. 6, Convolutional Pooling Layer is a dimensionality reduction algorithm that decreases the dimension of the feature matrix to make our network training more efficient. Besides, fully connected layer gets a huge number of parameters which is computationally inefficient [20]. In CNN, convolutional pooling is a down sampling technique used for image dimension reduction. There are many varieties of pooling layers like max-pooling, sum pooling, global pooling, average pooling, etc [19]. Pooling is the operation of downsampling the input feature that is provided to a layer. Max pooling looks for the most high-valued feature in a particular region and neglects all other remaining information (Fig. 7). The task of average pooling is near identical as max-pooling to reduce the image size of the given input also. When we apply max pooling we try to pick the most important high-valued feature and take it forward. The sum pooling takes the summation of the features in the given matrix. There is alternative variant of the pooling also known as global average pooling which we can use at the very end of the forward propagation. Global average pooling make use of an entire channel and finds out the median value of that channel instead of a particular region. The RGB value of image is passed on the convolutional layer which produces feature maps and then max pooling layer reduces dimensionality of the image (shown in Fig. 6). After than Fully Connected Layer takes place to make learn and make predictions. However, the filter used in the feature maps learns from the training. 


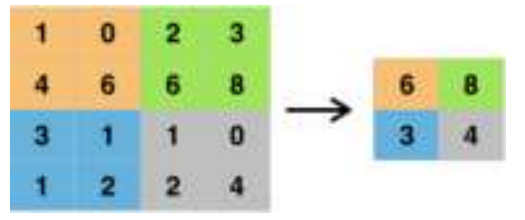

Fig. 7. 2x2 Max Pooling [28]

Convolutional Layer uses this type of filter or random filter values on runtime to extract feature maps from a given input image [19]. Below is the sample filter's representation from our desire MRI dataset,

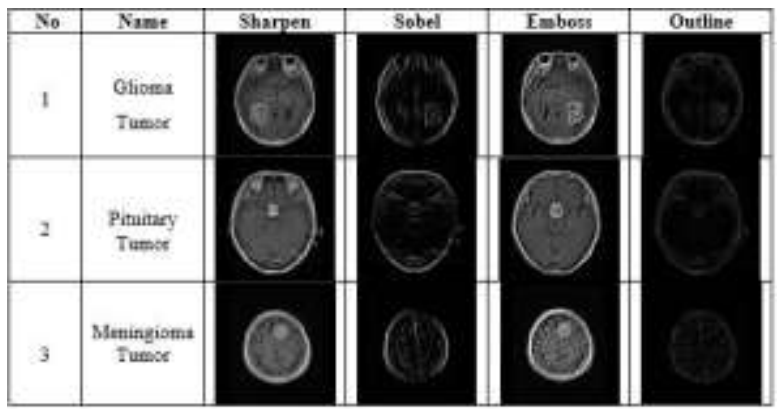

Fig. 8. Results after applying different types of Filters

After applying the filter's Sharpen, Sobel, Emboss and Outline in Fig. 8, we found the different structural changes in the image which is very important for a convolutional neural network. As we know that, convolutional neural networks learns from features sets applied by the filters.

\section{F. Transfer Learning}

Transfer learning is another machine learning mechanism where previously trained models are reused for a different deep learning problem. Transfer learning has become quite popular in the domain of image classification as well as natural language processing because of their fascinating predictive capabilities. Here, we are using a pre-trained transfer learning model then we try to retrain it for a new problem. Using transfer learning saves a lot of computation power because these pre-trained models which are trained on millions of images if we try to train that from the beginning it will put a price on time and computation power [14]. Transfer learning focuses on stored knowledge obtained while training and applied the knowledge to a different related problem. We can use pre-trained model knowledge of this visual world and transfer that knowledge into solving a different problem. For, Commercial purpose transfer learning works very well in reallife problems as it has the knowledge of millions of images for pattern detection. So, it becomes really good on image classification. These models are extremely data-intensive and depends on a big amount of labeled data to gain better performance [15]. We look to transfer as much knowledge as we can from the data source to our target data source or domain. Transfer learning is very helpful to deal with these innovative tasks and is important for a production scale that goes beyond domain-based tasks.

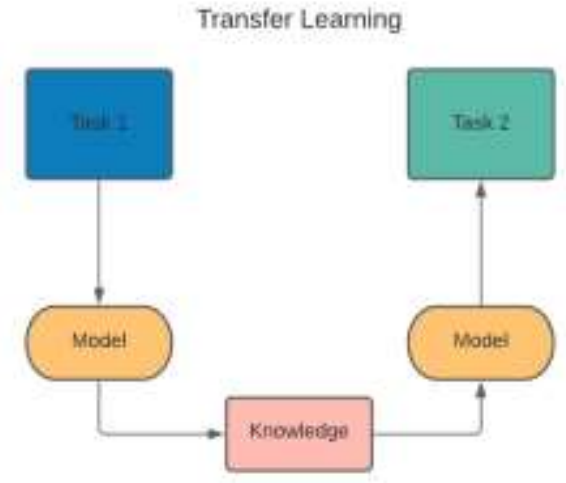

Fig. 9. Transfer Learning

To able to understand the concepts that transfer learning involves we have used pre-trained models like VGG16, EfficientNet-B3, ResNet-150V2, Inception-ResNetV2 to train our MRI Brain tumor classification problem for outstanding performance with less computational time and power.

\section{G. Temporal Convolutional Neural Network}

Temporal Convolutional Network is another variation of CNN for sequence or time series data modeling tasks. TCN performs better than GRU/LSTM architectures on huge number of tasks. Temporal Convolutional Networks has longer memory than other recurrent neural network including the same amount of capacity. TCN networks do not use synaptic gating mechanism and these networks have much longer memory [16]. The best feature this architecture provides that convolution are causal meaning there is no information leak from future to past and this network can handle any number of input and map it to an output of any sequence of same length. Temporal Convolutional Network make use of causal convolutions to map the convolution at time $\mathrm{t}$ elements that prior to previous layer. Temporal Convolutional Network uses 1-D convolutional network, where length of each of the hidden layer is same as input layer with same length of zero padding is also added make other layers same as previous ones [17]. Here we are using a TCN network to classify our MRI Brain images with an input size of $224 \times 224 \times 1=50176$. We are using dilations factors $d=\{1$, $2,4,8,16,32,64,128,256\}$ with a kernel size of 11 for classification task. Below is the network diagram of our temporal convolutional network.

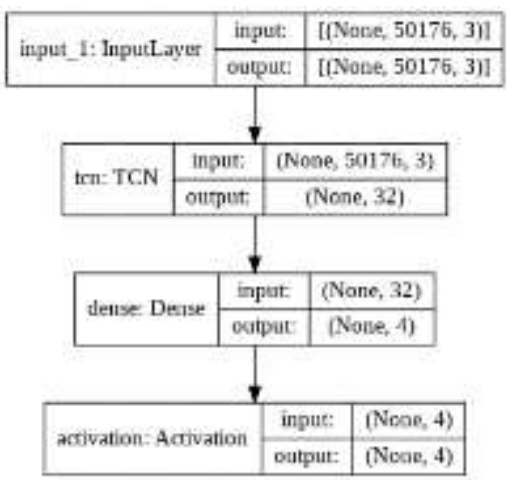

Fig. 10. TCN Network 
Temporal Convolutional Network has quite functional advantages like parallelism, flexible receptive filed size, stable gradients and low memory for training. TCN networks performs well because they do not face gradient exploding or gradient vanishing problems.

\section{RESEARCH METHODOLOGY}

The Research Methodology has three distinctive group: 1) Data Acquisition \& Distribution, 2) Image Pre-Processing \& Augmentation, 3) Training Models. Data Acquisition \& Distribution part is about the dataset and the property of the dataset it holds. Image Pre-Processing tasks is to pre-process the images for the training. Training part holds the information about all the models that we have used for research. Support Vector Machine (SVM), Random Forest (RF) and K-Nearest Neighbor (KNN) is used for comparing the Results found after the training. Convolutional Neural Networks (CNN) models and Transfer Learning's pre-trained weights are used for training the models specified in this paper.

\section{A. Data Acquisition \& Distribution}

From Kaggle, we have collected the dataset named "Brain Tumor Classification (MRI)". The dataset has total of 3264 MRI Brain Tumor images. The dataset has 2870 training images along with 394 testing images. Four distinct types of tumor images group is there in the dataset: Glioma Tumor, Meningioma Tumor, Pituitary Tumor and No Tumor. The dataset contains a total of 4 classes. Our work is to use different type's model to identify the tumor type. For this, we have distributed $80 \%$ image data for training and used $20 \%$ image data for validation. We have distributed training data in $80: 20$ ratio so that it can improve the model performance and increase the efficiency in detection.

\section{B. Image Pre-Processing \& Augmentation}

Before training phase starts, we need to pre-process the image so that the models we have used can learn accurately. The images that are collected is not in the same dimension that's why we have resized all the image in 224 x 224 dimension. Image Pre-Processing is needed to suppress the unwanted noise information and acquire clean data for training phase. Besides, after applying normalization training time substantially decreases and increases the model performance. Augmentation is responsible for generating new training examples out of existing data. It's nearly impossible to capture every outcome of real world scenario. Adjusting training data to can be helpful in predicting the type of tumors in wider array of possible outcomes. Used augmentations are: 1. Resizing (224 x 224), 2. Orientation (Horizontal \& Vertical), 3. Random Rotation $\left(30^{\circ}\right)$, 4. Zoom (0.3), 5. Width and Height Shift (0.2).

\section{Training Models}

In training phase, we have used the Convolutional Neural Network to extract feature maps from the image and this feature maps data is being forwarded to the selected Machine learning and Deep Learning algorithms. Traditional Machine Learning algorithms like Support Vector Machine (SVM), KNearest Neighbor (KNN), and Random Forest (RF) classifier are fused on the basis of majority voting [1]. These algorithms need features maps produced from the last layer of model (shown in Fig. 11). Then uses their individual classification technique for tumor detection [1]. Nevertheless, theses classifiers requires a huge amount of training time to produce accurate prediction.

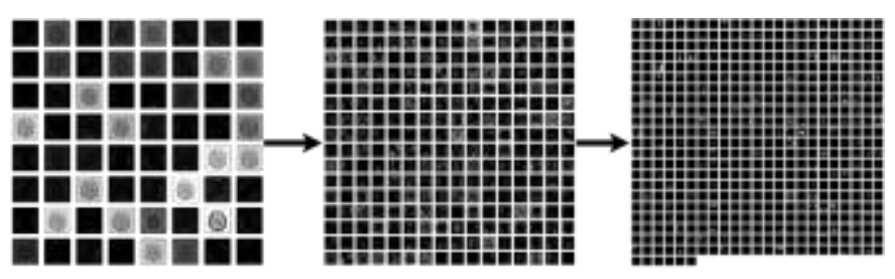

Fig. 11. Feature Maps of a single Brain tumor image from first convolution layer and last convolution layer

But in deep learning domain, the convolutional neural networks has much more advantage in image classification and detection tasks. Deep Learning models like EfficientNetB3, Resnet-150v2, Inception-Resnet-V2, Vgg16 and Temporal Convolutional Network (TCN) are used for tumor classification and the result is significantly good (shown in below Table-1). These models has been trained on "Imagenet" dataset with 1000 classes. Besides, these models gained knowledge about image filters. We have customized the input and output layer to re-train the models with our MRI Brain Tumor dataset with 4 classes. Feature map values are extracted from Convolutional Layer and forward the information to the densely connected network. Finally, the Adam optimization is applied to gain high accuracy for our deep learning models. While the training phase is running, validation accuracy is calculated respectively, and validation loss is also calculated in this process. Process of the work is showed on Fig. 12. The process shows data acquisition, preprocessing, augmentation and model training (Fig. 12).

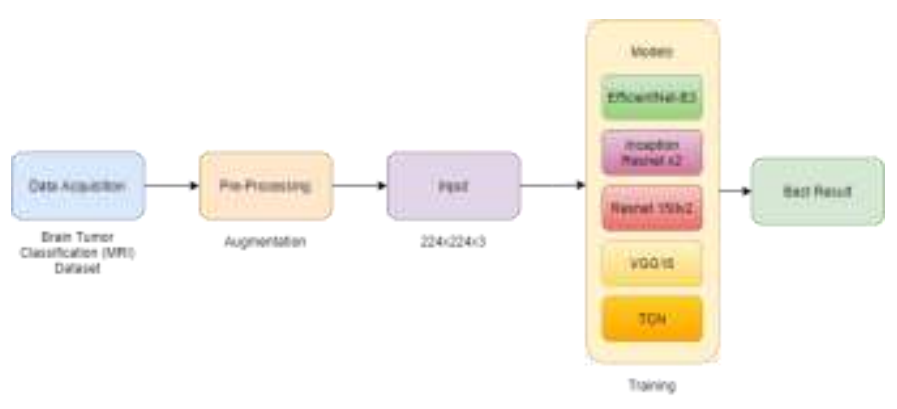

Fig. 12. Visualization of the Process

Efficient and accurate model is found via comparing accuracy and epochs. Model that has less epoch with higher accuracy is the efficient and the most performing one. In this MRI Brain Tumor classification, EfficientNet-B3 is the most performing model and has accuracy of $98.16 \%$. 


\section{Results ANd PERFormance EVAluation}

In this case study, we have found Convolutional Neural Network (CNN) Efficient Net B-3 performed better at MRI Brain Tumor classification. CNN is very good at image pattern recognition. Other, Machine learning algorithms performed well respectively but did not produce result as good as Convolutional Neural Networks. Support Vector Machine (SVM), K-Nearest Neighbor (KNN), Random Forest (RF) were good in the early days of Machine Learning. But Deep Learning method Convolutional Neural Network produced much more good results than other algorithms in a short amount of time. Thus, we need to use more Deep Learning algorithms for better MRI image recognition such that, Vgg16, EfficientNet-B3, ResNet-150V2, Inception ResNetV2, TCN etc. This research is based on the analysis of the results and the accuracy we found throughout our experiment.

Performance Evaluation:

True Positive $=\mathrm{TP}, \quad$ True Negative $=\mathrm{TN}$

False Positive $=$ FP,$\quad$ False Negative $=$ FN

1) Accuracy $=\frac{(T P+T N)}{(T P+F N+F P+T N)}$

2) Recall $=\frac{\llbracket(T p)}{(T P+F N)}$

3) Precision $=\frac{(T P)}{(T P+F P)}$

4) $\mathrm{F} 1=2 *\left(\frac{\text { Precision } x \text { Recall }}{\text { Precision }+ \text { Recall }}\right)$

A. Using Vgg16

Epoch vs. Accuracy:

Epochs rs. Training and Vatidation Ascuracy/loss
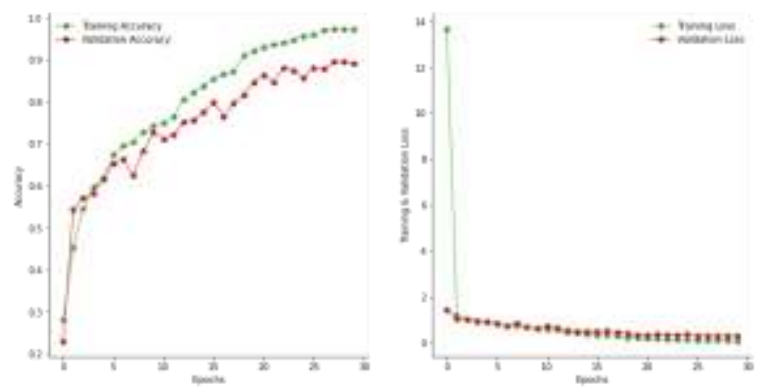

Fig. 13. Vgg16 Accuracy \& Loss

Confusion Matrix:

\begin{tabular}{|l|l|l|l|l|l|l|}
\hline Class & \multicolumn{4}{|l|}{ Assigned } & \multirow{2}{*}{ Name } \\
\hline \multirow{4}{*}{ Actual } & $\mathbf{0}$ & $\mathbf{1}$ & $\mathbf{2}$ & $\mathbf{3}$ & $\#$ & \\
\cline { 2 - 7 } & 79 & 2 & 12 & 0 & $\mathbf{0}$ & glioma_tumor \\
\cline { 2 - 7 } & 1 & 42 & 6 & 2 & $\mathbf{1}$ & no_tumor \\
\cline { 2 - 7 } & 5 & 4 & 85 & 2 & $\mathbf{2}$ & meningioma_tumor \\
\cline { 2 - 7 } & 1 & 1 & 3 & 82 & $\mathbf{3}$ & pituitary_tumor \\
\hline
\end{tabular}

Here Vgg16,

$$
\begin{aligned}
& \text { Accuracy }=\frac{(T P+T N)}{(T P+F N+F P+T N)}=88.07 \% \\
& \text { Recall } \quad=\frac{(T P)}{(T P+F N)}=0.88 \\
& \text { Precision }=\frac{(T P)}{(T P+F P)}=0.88 \\
& \mathrm{~F} 1 \quad=2 *\left(\frac{P \text { recision } x \text { Recall }}{\text { Precision }+ \text { Recall }}\right)=0.88
\end{aligned}
$$

Also, we have used around 30 epochs in the vgg 16 model and achieved $88.07 \%$ validation accuracy in average.

\section{B. Using EfficientNet-B3}

Epoch vs. Accuracy:

Epochs vs. Training and Vatidation Accuracy/loss
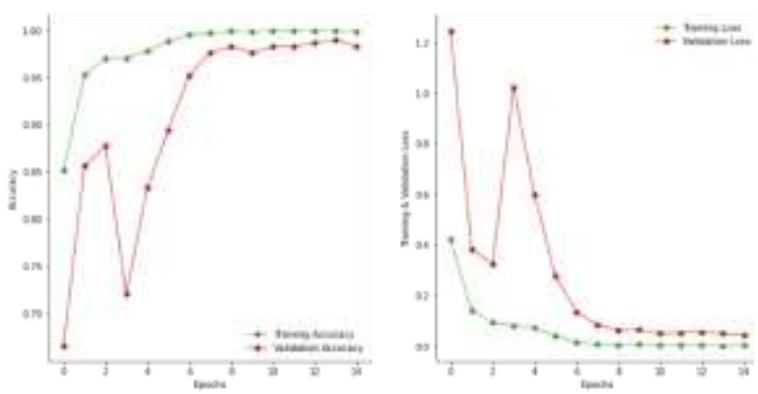

Fig. 14. EfficientNet-B3 Accuracy \& Loss

Confusion Matrix:

\begin{tabular}{|l|l|l|l|l|l|l|}
\hline Class & \multicolumn{4}{|l|}{ Assigned } & \multirow{2}{*}{ Name } \\
\hline \multirow{4}{*}{ Actual } & $\mathbf{0}$ & $\mathbf{1}$ & $\mathbf{2}$ & $\mathbf{3}$ & $\#$ & \\
\cline { 2 - 7 } & 89 & 2 & 1 & 0 & $\mathbf{0}$ & glioma_tumor \\
\cline { 2 - 7 } & 0 & 49 & 0 & 0 & $\mathbf{1}$ & no_tumor \\
\cline { 2 - 7 } & 1 & 0 & 95 & 0 & $\mathbf{2}$ & meningioma_tumor \\
\cline { 2 - 6 } & 0 & 0 & 2 & 87 & $\mathbf{3}$ & pituitary_tumor \\
\hline
\end{tabular}

Here EfficienNet-B3,

$$
\begin{aligned}
& \text { Accuracy }=\frac{(T P+T N)}{(T P+F N+F P+T N)}=98.16 \% \\
& \text { Recall }=\frac{(T P)}{(T P+F N)}=0.98 \\
& \text { Precision }=\frac{(T P\})}{(T P+F P)}=0.98 \\
& \text { F1 } \quad=2 *\left(\frac{P \text { recision } x \text { Recall }}{\text { Precision }+ \text { Recall }}\right)=0.98
\end{aligned}
$$

Well, in the EfficientNet-B3 model after 15 epochs, we have achieved validation accuracy around $98.16 \%$.

\section{Using Inception-ResNet-v2}

Epoch vs. Accuracy:

Epochs vs. Training and Volidation Accuracy/loss
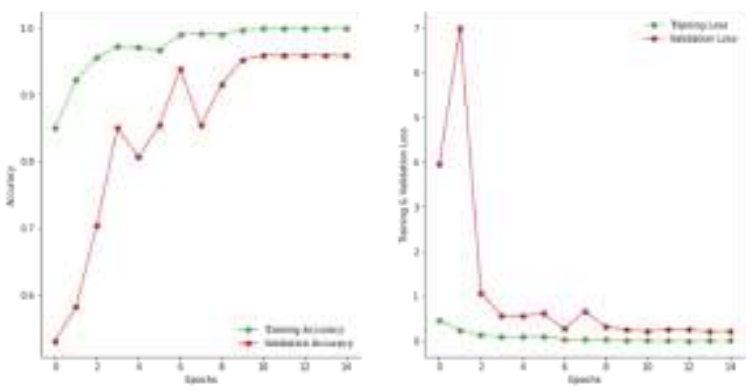

Fig. 15. Inception-Resnet-V2 Accuracy \& Loss 
Confusion Matrix:

\begin{tabular}{|l|l|l|l|l|l|l|}
\hline Class & \multicolumn{4}{|l|}{ Assigned } & \multirow{2}{*}{ Name } \\
\hline \multirow{4}{*}{ Actual } & $\mathbf{0}$ & $\mathbf{1}$ & $\mathbf{2}$ & $\mathbf{3}$ & $\#$ & \\
\cline { 2 - 7 } & 90 & 2 & 1 & 0 & $\mathbf{0}$ & glioma_tumor \\
\cline { 2 - 7 } & 0 & 48 & 0 & 3 & $\mathbf{1}$ & no_tumor \\
\cline { 2 - 7 } & 2 & 0 & 93 & 1 & $\mathbf{2}$ & meningioma_tumor \\
\cline { 2 - 6 } & 0 & 0 & 2 & 87 & $\mathbf{3}$ & pituitary_tumor \\
\hline
\end{tabular}

Here Inception-Resnet-v2,

Accuracy $=\frac{(T P+T N)}{[(T P+F N+F P+T N)}=96.66 \%$

Recall $=\frac{(T P)}{(T P+F N)}=0.97$

Precision $=\frac{\llbracket(T P)}{(T P+F P)}=0.97$

F1 $\quad=2 *\left(\frac{\text { Precision } x \text { Recall }}{\text { Precision }+ \text { Recall }}\right)=0.97$

Moreover, Inception-Resnet-v2 got an accuracy of $96.66 \%$ with a number of 15 epoch.

D. Using Resnet-150-v2

Epoch vs. Accuracy:

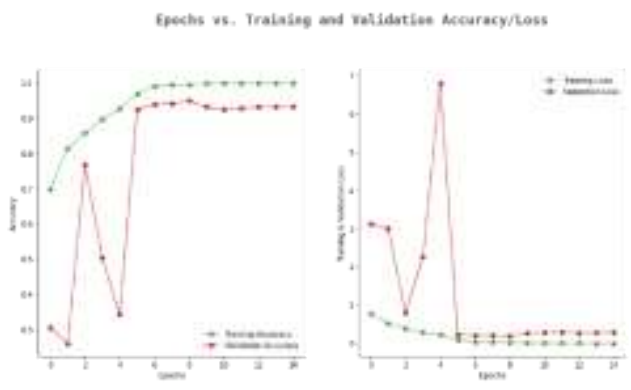

Fig. 16. Resnet-150v2 Accuracy \& Loss

Confusion Matrix:

\begin{tabular}{|l|l|l|l|l|l|l|}
\hline Class & \multicolumn{4}{|l|}{ Assigned } & \multirow{2}{*}{ Name } \\
\hline \multirow{4}{*}{ Actual } & $\mathbf{0}$ & $\mathbf{1}$ & $\mathbf{2}$ & $\mathbf{3}$ & $\#$ & \\
\cline { 2 - 7 } & 87 & 3 & 3 & 0 & $\mathbf{0}$ & glioma_tumor \\
\cline { 2 - 7 } & 0 & 50 & 1 & 0 & $\mathbf{1}$ & no_tumor \\
\cline { 2 - 7 } & 1 & 1 & 92 & 2 & $\mathbf{2}$ & meningioma_tumor \\
\cline { 2 - 7 } & 1 & 0 & 0 & 86 & $\mathbf{3}$ & pituitary_tumor \\
\hline
\end{tabular}

Here Resnet-150v2,

Accuracy $=\frac{(T P+T N)}{(T P+F N+F P+T N)}=95.74 \%$

Recall $=\frac{(T P)}{(T P+F N)}=0.96$

Precision $=\frac{\llbracket(T P)}{(T P+F p)}=0.96$

F1 $\quad=2 *\left(\frac{\text { Precision } x \text { Recall }}{\text { Precision }+ \text { Recall }}\right)=0.96$

After 15 epochs, Resnet-150v2 models validation accuracy is gone flat and therefore stopped the model for further training. At this point, Resnet-150v2 the model got $95.74 \%$ accuracy in average.

\section{E. Using TCN}

Epoch vs. Accuracy:
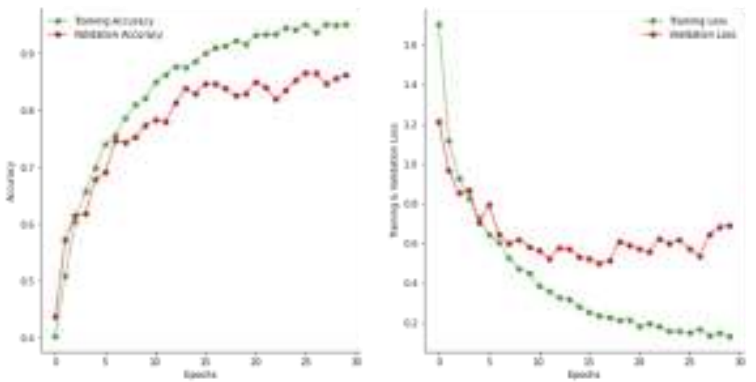

Fig. 17. TCN Accuracy \& Loss

Confusion Matrix:

\begin{tabular}{|l|l|l|l|l|l|l|}
\hline Class & \multicolumn{4}{|l|}{ Assigned } & \multirow{2}{*}{ Name } \\
\hline \multirow{4}{*}{ Actual } & $\mathbf{0}$ & $\mathbf{1}$ & $\mathbf{2}$ & $\mathbf{3}$ & $\#$ & \\
\cline { 2 - 7 } & 83 & 0 & 13 & 1 & $\mathbf{0}$ & glioma_tumor \\
\cline { 2 - 7 } & 2 & 37 & 7 & 2 & $\mathbf{1}$ & no_tumor \\
\cline { 2 - 7 } & 11 & 4 & 85 & 1 & $\mathbf{2}$ & meningioma_tumor \\
\cline { 2 - 6 } & 1 & 0 & 3 & 77 & $\mathbf{3}$ & pituitary_tumor \\
\hline
\end{tabular}

Here TCN,

$$
\begin{aligned}
& \text { Accuracy }=\frac{(T P+T N)}{(T P+F N+F P+T N)}=85.46 \% \\
& \text { Recall } \quad=\frac{(T P)}{(T P+F N)}=0.86 \\
& \text { Precision }=\frac{(T P)}{(T P+F P)}=0.86 \\
& \mathrm{~F} 1 \quad=2 *\left(\frac{P \text { recision } x \text { Recall }}{\text { Precision }+ \text { Recall }}\right)=0.86
\end{aligned}
$$

TCN network ran for 30 epoch and achieved $85.46 \%$ accuracy

\begin{tabular}{|c|c|c|c|c|c|}
\hline $\begin{array}{l}\text { Algorithm } \\
\text { s }\end{array}$ & Labels & Precision & Recall & F1 & $\begin{array}{c}\text { Accuracy } \\
(\%)\end{array}$ \\
\hline \multirow{3}{*}{ SVM [1] } & Glioma & 85 & 85 & 87 & \multirow{3}{*}{$79.9 \%$} \\
\hline & Meningioma & 76 & 64 & 69 & \\
\hline & Pituitary & 97 & 94 & 92 & \\
\hline \multirow{3}{*}{ KNN [1] } & Glioma & 91 & 93 & 92 & \multirow{3}{*}{$91.83 \%$} \\
\hline & Meningioma & 88 & 83 & 85 & \\
\hline & Pituitary & 97 & 97 & 98 & \\
\hline \multirow{3}{*}{$\mathrm{RF}[1]$} & Glioma & 86 & 83 & 85 & \multirow{3}{*}{$77.87 \%$} \\
\hline & Meningioma & 80 & 55 & 66 & \\
\hline & Pituitary & 95 & 88 & 91 & \\
\hline $\begin{array}{l}\text { EFFICIENT } \\
\text { NET-B3 }\end{array}$ & Glioma & 99 & 96 & 97 & $98.16 \%$ \\
\hline
\end{tabular}
which is still better than some of the machine learning algorithms.

TABLE 1. Performance Metrics 


\begin{tabular}{|c|c|c|c|c|c|}
\hline & Meningioma & 98 & 99 & 98 & \\
\hline & Pituitary & 98 & 100 & 99 & \\
\hline \multirow{3}{*}{$\begin{array}{l}\text { RESNET- } \\
\text { 150v2 }\end{array}$} & Glioma & 98 & 94 & 96 & \multirow{3}{*}{$95.74 \%$} \\
\hline & Meningioma & 96 & 96 & 96 & \\
\hline & Pituitary & 98 & 99 & 98 & \\
\hline \multirow{3}{*}{$\begin{array}{l}\text { INCEPTION } \\
\text {-RESNETV2 }\end{array}$} & Glioma & 98 & 97 & 97 & \multirow{3}{*}{$96.66 \%$} \\
\hline & Meningioma & 99 & 97 & 98 & \\
\hline & Pituitary & 96 & 100 & 98 & \\
\hline \multirow{3}{*}{ VGG16 } & Glioma & 92 & 85 & 88 & \multirow{3}{*}{$88.07 \%$} \\
\hline & Meningioma & 80 & 89 & 84 & \\
\hline & Pituitary & 95 & 94 & 95 & \\
\hline \multirow{3}{*}{ TCN } & Glioma & 86 & 86 & 86 & \multirow{3}{*}{$85.46 \%$} \\
\hline & Meningioma & 79 & 84 & 81 & \\
\hline & Pituitary & 95 & 95 & 95 & \\
\hline
\end{tabular}

Precision, recall \& F1: macro-averaged (equally weighted avg. of 4 classes).

From Table. 1, we have perceived the accuracy of Support Vector Machine (SVM) is 79.9\%, K-Nearest Neighbor (KNN) is $91.83 \%$, and Random Forest (RF) is $77.87 \%$ from recent studies [1]. In our pre-trained model, we acquired accuracy level of $98.16 \%$ in EfficientNet-B3, 96.66\% in InceptionResNet-v2, 95.74\% in ResNet-150v2, 88.07\% in VGG16 and $85.46 \%$ in Temporal Convolutional Network (TCN) [26]. Besides, individual score for precision, recall and $\mathrm{F} 1$ is calculated for each classes like Glioma, Meningioma and Pituitary Tumor. We can clearly see that Deep Learning models like EfficientNet-B3 [23], Inception-Resnetv2 [24, 25] and Resnet150v2 [24] is clearly better than other models introduced in the paper. Above all, EfficientNet-B3 classifier achieved higher accuracy amongst all other models.

\section{DISCUSSION}

To figure out the early stage of brain tumor, we need an efficient MRI brain tumor classification for our medical therapy. So, considering our current medical tools, we can treat individuals before it's too late. The main research is to discover a brain tumor classifier with high accuracy and performance. In the traditional image recognition of the brain, classification is carried out by using image segmentation. The

complexity is lower than the other networks. Computation time is high, and the accuracy seems too low. The model's accuracy development is leading to a very demanding compensated sector. From SVM, RF, DT, KNN, and CNN algorithms convolutional neural networks model EfficientNetB3 [23] performed well in the image feature recognition of Brain Tumor Detection. So, we can utilize the use of tumor identification using these Convolutional Neural Networks to get high accuracy in less amount of time. Thus, we prefer selecting convolutional neural networks like EfficientNetB3 for brain tumor identification.

\section{CONCLUSION}

Brain is vital portion of human body which controls the overall activity of human body and maintain all the functionality. So, it is very much important to keep brain away from any harm. That is why tumor detection is very much important and has to be detected as soon as possible. By the grace of technology, we can improve the detection process by using different machine and deep learning algorithms. In this paper, different algorithms are introduced and studied for finding the better classification process. At the end, CNN is found most suitable deep learning method. Through Convolutional Layer and Max Pooling, feature maps are being extracted and get trained with a fully connected dense neural network. After training, we can classify MRI images with given labels attached to them. Whether the brain has Benign and Malignant tumors, we can detect with given MRI images. At this point, we found that the Deep Learning method CNN model EfficientNet-B3 performed very well at large-scale image pattern recognition.

\section{REFERENCES}

[1] Neelum Noreen1, Sellapan Palaniappan1, Abdul Qayyum2, Iftikhar Ahmad3 and Madini O. Alassaf "Brain Tumor Classification Based on Fine-Tuned Models and the Ensemble Method" Vol.67, No.3, pp. 39673982, 2021

[2] M. Sajjad, S. Khan, K. Muhammad, W. Wu, A. Ullah et al., "Multi-grade brain tumor classification using deep CNN with extensive data augmentation," Journal of Computational Science, vol. 30, pp. 174- 182, 2019

[3] El-Dahshan, E.S.A., Hosny, T., Salem, A.B.M., "Hybrid intelligent techniques for MRI brain images classification", Digital Signal Processing, Elsevier, vol. 20, no. 2, pp.433-441, 2010.

[4] A. Sengur, "An expert system based on principal component analysis, artificial immune system and fuzzy k-NN for diagnosis of valvular heart diseases", Comp. Biol. Med. (2007).

[5] M. O'Farrell, E. Lewis, C. Flanagan, N. Jackman, "Comparison of k-NN and neural network methods in the classification of spectral data from an optical fibre-based sensor system used for quality control in the food industry", Sens. Actuators B: Chemical 111-112C (2005) 354-362.

[6] Maitra, M., Chatterjee, A., Matsuno, F., "A novel scheme for feature extraction and classification of magnetic resonance brain images based on plantlet transform and support vector machine", In Proceedings of International Conference on Instrumentation, Control and Information Technology, pp.1130-1134, 2008.

[7] G. Singh and M. Ansari, "Efficient detection of brain tumor from MRIs using K-means segmentation and normalized histogram," in 2016 1st India Int. Conf. on Information Processing, Delhi, India, pp. 1- 6, 2016.

[8] Provost F, Hibert C, Malet J P, et al. "Automatic classification of endogenous seismic sources within a landslide body using random forest algorithm" [C]//EGU General Assembly Conference Abstracts. 2016, 18: 15705. 
[9] Qiong Ren, Hui Cheng, Hai Han, "Research on Machine Learning Framework Based on Random Forest Algorithm", AIP Conference Proceedings 1820, 080020 (2017).

[10] Prachi Damodhar Shahare, Ram Nivas Giri, "Comparative Analysis of Artificial Neural Network and Support Vector Machine Classification for Breast Cancer Detection", International Research Journal of Engineering and Technology (IRJET), vol-02, issue-09,Dec 2015.

[11] Rohith Gandhi, "Support Vector Machine- Introduction to Machine Learning Algorithm", 2018.

[12] M. W. Libbrecht and W. S. Noble, "Machine learning applications in genetics and genomics," Nature Reviews Genetics, vol. 16, no. 6, pp. 321-332, 2015.

[13] S. S. Nikam, "A comparative study of classification techniques in data mining algorithms," Oriental journal of computer science \& technology, vol. 8, no. 1, pp. 13-19, 2015.

[14] Jason Brownlee, “ A Gentle Introduction to Transfer Learning for Deep Learning”, 2017.

[15] Hoo-Chang Shin, Holger R. Roth, Mingchen Gao, Le Lu, Ziyue Xu, Isabella Nogues, Jianhua Yao, Daniel Mollura, and Ronald M. Summers, "Deep Convolutional Neural Networks for Computer-Aided Detection: CNN Architectures, Dataset Characteristics and Transfer Learning", IEEE Trnasctions on Medical Imaging, vol. 35, no. 5, MAY 2016

[16] Colin Lea, Michael D. Flynn, Rene Vidal, Austin Reiter, Gregory D. Hager, "Temporal Convolutional Networks for Action Segmentation and Detection", 2016.

[17] Yangdong He, Jiabao Zhao, "Temporal Convolutional Networks for Anomaly Detection in Time Series", Journal of Physics: Conference series, vol-1213, issue-4, 2019.

[18] Badža MM, Barjaktarović MC (2020), "Classification of brain tumors from MRI images using a convolutional neural network", Appl Sci 10(6):1-13.

[19] Çinar A, Yildirim M (2020), "Detection of tumors on brain MRI images using the hybrid convolutional neural network architecture", Med Hypotheses 139:109684.

[20] Z. N. K. Swati, Q. Zhao, M. Kabir, F. Ali, Z. Ali et al., "Brain tumor classification for MR images using transfer learning and fine-tuning," Computerized Medical Imaging and Graphics, vol. 75, pp. 34-46, 2019.

[21] M. D. Zeiler and R. Fergus, "Visualizing and understanding convolutional networks," in European conference on computer vision, 2014, pp. 818833.

[22] K. Simonyan and A. Zisserman, "Very deep convolutional networks for large-scale image recognition," arXiv preprint arXiv:1409.1556, 2014.

[23] Mingxing Tan, Quoc V. Le. ,"EfficientNet: Rethinking Model Scaling for Convolutional Neural Networks".

[24] Kaiming He Xiangyu Zhang Shaoqing Ren Jian Sun , "Deep Residual Learning for Image Recognition", Microsoft Research.

[25] Christian Szegedy, Sergey Ioffe, Vincent Vanhoucke, Alex Alemi , "Inception-v4, Inception-ResNet and the Impact of Residual Connections on Learning".

[26] Colin Lea, Rene Vidal, Austin Reiter, Gregory D. Hager, "Temporal Convolutional Networks: A Unified Approach to Action Segmentation".

[27] Karen Simonyan, Andrew Zisserman, "Very Deep Convolutional Networks for Large-Scale Image Recognition".

[28] http://deepai.org/machine-learning-glossary-and-terms/max-pooling

[29] https://yashuseth.blog/2018/02/11/which-activation-function-to-use-inneural-networks/

[30] https://zhuanlan.zhihu.com/p/35146770

[31] https://builtin.com/data-science/random-forest-algorithm

[32] https://www.hopkinsmedicine.org/health/conditions-and-diseases/braintumor/brain-tumor-types

[33] https://www.cancercenter.com/cancer-types/brain-cancer/types

[34] https://morioh.com/p/2993724711d8

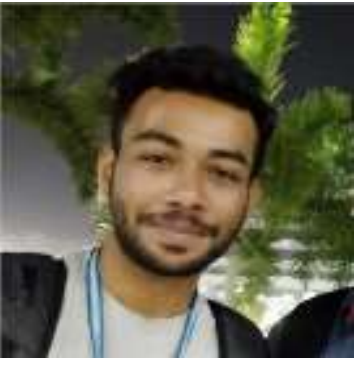

Partha Sutradhar ${ }^{1}$ was born in Dhaka, Bangladesh on August 02, 1998. He is also pursuing his Bachelor of Science (BSc) Degree in Computer Science and Engineering (CSE) from American International UniversityBangladesh (AIUB). In 2020, He worked as a Student Assistant in AIUB Software Division Department. He is also looking for a post-graduation degree in Software Engineering. $\mathrm{He}$ is specialized in System Architecture, Embedded System and Deep Learning. His region of interest includes Artificial Intelligence, Computer Vision, Automation, IoT, Neural Network Optimization, Image Processing and, Embedded Systems.

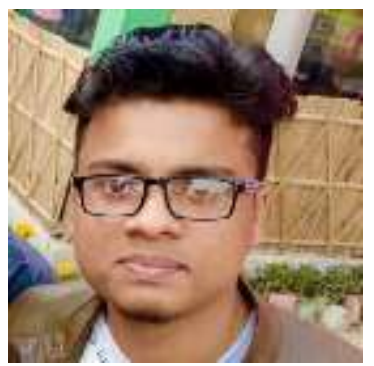

Prosenjit Kumer Tarefder ${ }^{2}$ was born in Madhupur, Tangail, Bangladesh on June 03, 1998. He completed his Bachelor of Science (BSc) degree in Computer Science and Engineering (CSE) from American International University-Bangladesh (AIUB). He completed his internship as a Teaching Assistant (TA) in "Computer Graphics" course offered by Faculty of Science and Technology, AIUB. He is also a professional graphics designer. His research interest and passion include Graphics Design, Artificial Intelligence, Image Processing and Computer Networks.

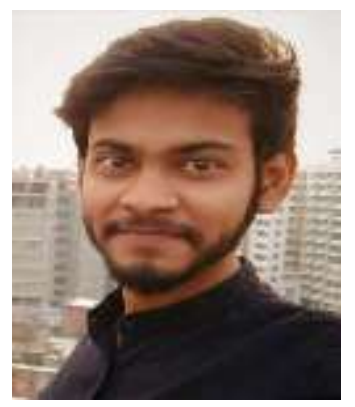

Imran Prodan ${ }^{3}$ was born in Mirpur, Dhaka, Bangladesh on May 05, 1998. He completed his Bachelor of Science (BSc) degree in Computer Science and Engineering (CSE) from American International University-Bangladesh (AIUB). He completed his internship as a Teaching Assistant (TA) in "Data Structure" course offered by Faculty of Science and Technology, AIUB.

His research interest and passion include Software Quality Assurance, Artificial Intelligence, Image Processing, and Computer Network.

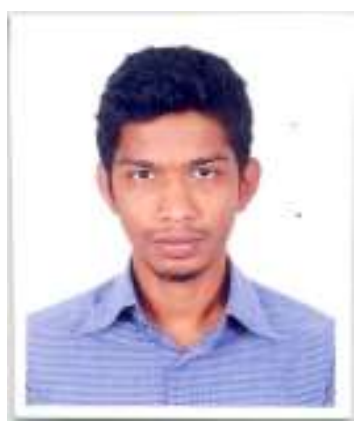

MD. Sheikh Saddi ${ }^{4}$ was born in Bhola, Barisal, Bangladesh on December 31, 1999. He is currently pursuing his Bachelor of Science (BSc) Degree in Computer Science and Engineering (CSE) from American International University Bangladesh (AIUB). In 2021, he joined as a Teaching Assistant (TA) at American International University Bangladesh (AIUB) and 
completed in the same year. He is also looking for a master degree in computer science.

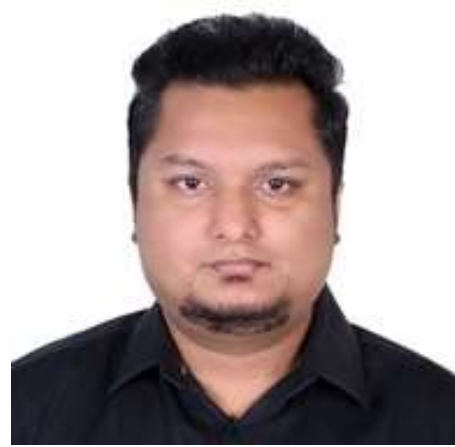

Victor Stany Rozario ${ }^{5}$ completed B.Sc. in

Computer Science \& Engineering and M.Sc.

in Computer Science from American International University-Bangladesh,

Dhaka, Bangladesh. Currently he is working as an Assistant Professor in the Department of Computer Science under the Faculty of Science and Technology, AIUB. His current research interest includes Data Science, Data Mining, Intelligent Systems, Machine Learning, Web Mining and Human Computer Interaction 